\title{
HOE for clock distribution in integrated circuits : Experimental results
}

\author{
D. Prongué and H. P. Herzig \\ University of Neuchâtel, Institute of Microtechnology, CH-2000 Neuchâtel, Switzerland
}

\begin{abstract}
This paper reports the design and fabrication of transmission holographic optical elements (HOEs) for clock distribution. First, we have studied and fabricated a multi-focus doublet HOE. The aberrations due to the wavelength shift between recording $(\lambda=488 \mathrm{~nm})$ and reconstruction $(\lambda=780 \mathrm{~nm})$ have been minimized by an appropriate recording and readout geometry. The diffraction efficiency has been optimized by a copying technique. Second, we have investigated the near-field internal reflection (TIR) holographic recording technique to solve the problems of miniaturization. With this method, we have recorded a $100 \times 100$ lenšlet array with focal lengths of $f=400 \mu \mathrm{m}$.
\end{abstract}

\section{INTRODUCTION}

Optical interconnection, using free-space light propagation, allows parallel communication, eliminates mechanical point to point contacts and has the potential of addressing any location on the integrated circuit. Holographic optical elements (HOE) are well suited to realize the required multiple imaging. This paper presents the design and fabrication of transmission HOEs for clock distribution. The basic set-up consists of a signal source (semiconductor laser) and a HOE, which images the source onto photodetectors in the integrated circuit.

The requirements for the HOEs are: low aberrations, low geometrical distortions and high efficiency. In addition, the method to construct the HOEs should be suitable for large numbers of interconnections. Different HOE recording methods will be discussed and some of them are tested experimentally.

Perfect 2-dimensional HOE structures can be generated by computer. To get high efficiencies, however, the computer generated holograms (CGH) have to be transformed into volume or surface-relief HOEs. Problems occur for high spatial frequencies, where Bragg diffraction or blazed structures are difficult to achieve properly. On the other hand, interferometric recording techniques suffer from aberrations caused by the wavelength shift between recording and reconstruction, imposed by the spectral sensitivity of the hologram recording material. Analytical equations, as well as a ray-tracing program have been applied to analyze the geometrical aberrations. The intensity distribution in the image plane has been calculated with the aid of the Rayleigh-Sommerfeld integral. Multiple step recording techniques or CGH wavefront generators can be used to simultaneously compensate the aberrations and fulfill the conditions for high efficiency. However, these techniques are not necessary for small NA and short focal lengths. We have found nearly diffraction limited spot sizes by optimizing the recording geometry of the HOEs.

First, a prototype for clock distribution onto a readily available photodetector array (Fig.2) has been fabricated ${ }^{1}$. The two elements of the doublet HOE are a collimator and a $5 \times 5$ multifacet HOE for multiple focussing. This doublet HOE has small aberrations and is insensitive to wavelength variations of the source. The holograms have been recorded at $\lambda=488 \mathrm{~nm}$ in silver halide emulsions, copied and then 
bleached. When reconstructed at $\lambda=780 \mathrm{~nm}$, this doublet HOE produces diffraction limited spots of $10 \mu \mathrm{m}$ diameter. Second, we have investigated the problems of miniaturization. For that purpose, a special test chip was realized at the CSEM (Centre Suisse d'Electronique et de Microtechnique) using CMOS technology and the near-field total internal reflection (TIR) holographic recording technique was introduced. By the same technique a holographic lenslet array with 10'000 interconnection points has been fabricated.

\section{HOE DESIGN}

The wavelength shift between recording and reconstruction leads to aberrations. As our HOEs are volume holograms, the wavelength shift may also reduce the diffraction efficiency, when the Bragg condition is no longer fulfilled. So, our main goal will be to maintain low aberrations and high diffraction efficiency.

\subsection{Analytical equations for the aberrations}

The principle parameters (recording and reconstructing angles, astigmatic focal lengths, third order terms) for a HOE can be calculated analytically ${ }^{1,2}$. In this paper, we shall limit ourselves to wavevectors lying in the same plane of incidence, and to plane reference waves $\left(\rho_{r}=\rho_{R}=\infty\right)$, as shown in Fig. 1. The direction $\theta_{\mathrm{P}}$ of the outgoing wavevector $\mathrm{k}_{\mathrm{P}}$ is given by the grating equation

$$
\sin \theta_{\mathrm{P}}=\sin \theta_{\mathrm{r}} \pm \mathrm{m} \mu\left(\sin \theta_{\mathrm{O}}-\sin \theta_{\mathrm{R}}\right)
$$

where $\mathrm{m}$ is the diffraction order ( $\mathrm{m}=+1$ in our case), $\mu=\lambda_{\mathrm{T}} \lambda_{\mathrm{R}}$, and $\theta_{\mathrm{i}}$ are the angles of incidence (Fig.1). The indices refer to the waves involved, namely $\mathrm{R}$ to the recording reference, $\mathrm{O}$ to the object point, $\mathrm{r}$ to the reconstructing reference and $\mathrm{P}$ to the primary reconstructed wave.

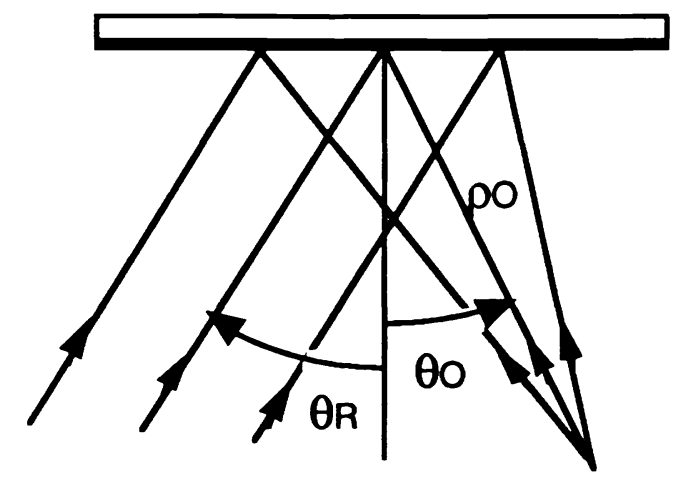

Fig.1 a) HOE recording geometry

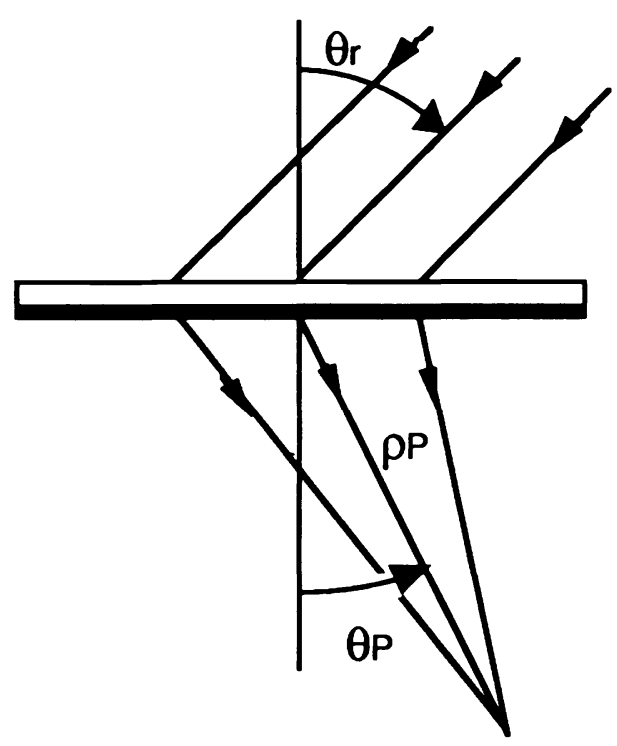

b) HOE readout geometry.

The principal curvatures of the astigmatic outgoing wave ${ }^{2}$ are described by 


$$
\frac{1}{\rho_{\mathrm{P}}{ }^{11}}=\mu\left(\frac{\cos \theta_{\mathrm{O}}}{\cos \theta_{\mathrm{P}}}\right)^{2} \frac{1}{\rho_{\mathrm{O}}}, \quad \frac{1}{\rho_{\mathrm{P}}{ }^{\perp}}=\mu \frac{1}{\rho_{\mathrm{O}}},
$$

where $\rho_{i}$ are the radii of curvature measured from the hologram, with $\rho_{r}=\rho_{R}=\infty$. The curvature of the astigmatic outgoing wave is given by the principal radii $\rho_{\mathrm{p}}{ }^{\prime \prime}$ and $\rho_{\mathrm{P}}{ }^{\mathrm{r}}$, parallel and perpendicular to the plane of incidence, respectively. The outgoing wave is only anastigmatic $\left(\rho_{p}{ }^{\prime \prime}=\rho_{p}{ }^{\perp}\right)$, if $\theta_{O}=\theta_{p}$. Thus, we will keep the angles of the object wave and the image wave equal, in order to eliminate the astigmatic aberration.

With $\rho_{P}=\rho_{P} \|=\rho_{P}{ }^{\perp}=\rho o / \mu$, the third order terms become

$$
\begin{aligned}
& c_{x x y}=\frac{1}{\cos \theta_{P}}\left(\frac{1}{\rho_{P}^{2}} \sin \theta_{P}-\frac{\mu}{\rho_{O}^{2}} \sin \theta_{O}\right), \\
& c_{y y y}=\frac{3}{2 \cos ^{3} \theta_{P}} \times\left(\frac{1}{\rho_{P}^{2}} \sin \theta_{P} \cos ^{2} \theta_{P}-\frac{\mu}{\rho_{O}^{2}} \sin \theta_{O} \cos ^{2} \theta_{O}\right), \\
& c_{x x x}=0, \quad c_{x y y}=0 .
\end{aligned}
$$

The coefficients $\mathrm{c}_{\mathrm{ijk}}$ describe the coma ${ }^{3}$. Note, that the $\mathrm{c}_{\mathrm{ijk}}$ disappear for a perfect spherical wave. In the off-axis case with conserved geometry $\left(\theta_{\mathrm{P}}=\theta_{\mathrm{O}} \neq 0^{\circ}\right)$, there is no astigmatism, but there will be coma. In the on-axis case $\left(\theta_{\mathrm{P}}=\theta_{\mathrm{O}}=0^{\circ}\right)$, coma disappears also.

\subsection{Numeric analysis of the aberrations}

The analytical equations for wave aberrations allow to determine a suitable geometry, for which the second and third order aberrations are cancelled. But higher order aberrations, more cumbersome to calculate analytically, can still be significant. For this reason, we have used a ray-tracing program. The results are typically presented as spot diagrams (Fig.5). The intensity distribution in the image plane has been calculated with the aid of the Rayleigh-Sommerfeld integral (Fig.6). These computer simulations have shown that cancelling second and third order aberrations was in our case sufficient to get nearly diffraction limited spots, because of the small focal length and aperture.

\subsection{Diffraction efficiency}

Besides low aberrations of the image wave, the efficiency of the HOE is important. To get high diffraction efficiency, we have to record a volume grating $\mathrm{HOE}$ and to fulfill the Bragg condition ${ }^{6}$. The problem is now, that the condition for low aberrations, described above, and maintaining the Bragg condition for a wavelength shift between recording and reconstruction, are not compatible.

Several methods have been proposed in the literature $3,4,5$ to satisfy the two conditions more or less simultaneously. They usually involve several steps for the HOE recording, where beams emerge from CGHs or intermediate HOEs. We have opted for a copying technique ${ }^{1}$. In the first step, an intermediate HOE, optimized for low aberrations after the wavelength shift, is recorded. Then, in the second step, this 
intermediate HOE is contact copied with another reference beam angle, which allows to adjust the fringe tilt in the new HOE to fulfill the Bragg condition in the center of the facets, while preserving the twodimensional structure of the fringes.

\section{DOUBLET HOE FOR INTERCONNECTION}

\subsection{Geometrical configuration}

For clock distribution, i.e. to focus one source to several locations, a multi-facet HOE is suitable. Each holographic facet contributes to the reconstruction of one single focus point, therefore no intermodulation occurs. Geometrical commodities favor a transmission hologram parallel to the detector plane.

An on-axis geometry $\left(\theta_{O}=0^{\circ}\right)$ would have the advantage to eliminate third order wave aberrations, while an off-axis geometry (higher spatial frequency) would have higher diffraction efficiency. Both advantages can be brought together by a doublet HOE composed of a collimating HOE and a focusing multi-facet HOE (Fig.2). The two components of the doublet HOE are free of aberrations up to third order, because the divergent source and the convergent image waves are both on-axis, whereas the plane reference waves are off-axis, as desired for high diffraction efficiency.

Pairs of HOEs have been recorded in order to get the following characteristics for the final doublet HOE: readout wavelength $\lambda_{r}=780 \mathrm{~nm}$, collimating focal length $f_{1}=50 \mathrm{~mm}$, focusing focal length $f_{2}=31 \mathrm{~mm}$, source wave on-axis $\left(\theta_{\mathrm{P} 1}=0^{\circ}\right)$, image wave on-axis $\left(\theta_{\mathrm{P} 2}=0^{\circ}\right)$, plane reference waves at $\theta_{\mathrm{r}}=30^{\circ}$, spacing between the HOEs $\mathrm{d}=0 \mathrm{~mm}$.

\subsection{HOE recording}

First, a pair of intermediate HOEs (collimating and focusing HOEs) has been recorded at $\lambda_{\mathrm{R}}=488 \mathrm{~nm}$ on silver halide plates (AGFA 8E56). The geometrical characteristics were: plane reference wave $\left(\rho_{R}=\infty\right)$ at $\theta_{R}=18.2^{\circ}$, object wave on-axis $\left(\theta_{O}=0^{\circ}\right)$ and spherical with $\rho_{O 1}=80 \mathrm{~mm}$ for the collimating and $\rho_{\mathrm{O} 2}=50 \mathrm{~mm}$ for the focusing intermediate $\mathrm{HOE}$. The $5 \times 5$ facet array of the focusing intermediate $\mathrm{HOE}$ has been recorded by step and repeat.

Both intermediate HOE have been contact copied at $\lambda_{R}=488 \mathrm{~nm}$, with a new plane reference wave at $\theta_{R}^{\prime}=24.1^{\circ}$. At this new reference wave angle the Bragg condition is not satisfied for the intermediate $\mathrm{HOE}$, but the diffraction efficiency is sufficient to record the new HOE.

\subsection{Experimental results and simulations}

The two HOE were sealed together and illuminated with a laser diode at $\lambda=780 \mathrm{~nm}$, as sketched in Fig.2. A picture of the prototype is shown in Fig.3. The reconstructed spots were analyzed using a microscope with a CCD camera mounted on a 3-D translation stage.

Figure 4 shows the center spot and a corner spot generated by the doublet HOE, when illuminated with a laser diode (Hitachi HL7801). The ray-tracing simulation for the same two spots produces the diagrams shown in Fig.5. The diffraction limit, imposed by the aperture of the focusing facets, was calculated approximately as $r \cong \lambda f_{2} / a=8.1 \mu \mathrm{m}$, (focal length $f_{2}=31 \mathrm{~mm}$, facet size $a=3 \mathrm{~mm}, \lambda=780 \mathrm{~nm}$ ) 
and is shown as circles in Fig.5. The intensity distribution in the image plane for the same two spots has been simulated with the aid of the Rayleigh-Sommerfeld integral. The results are shown in Fig.6.

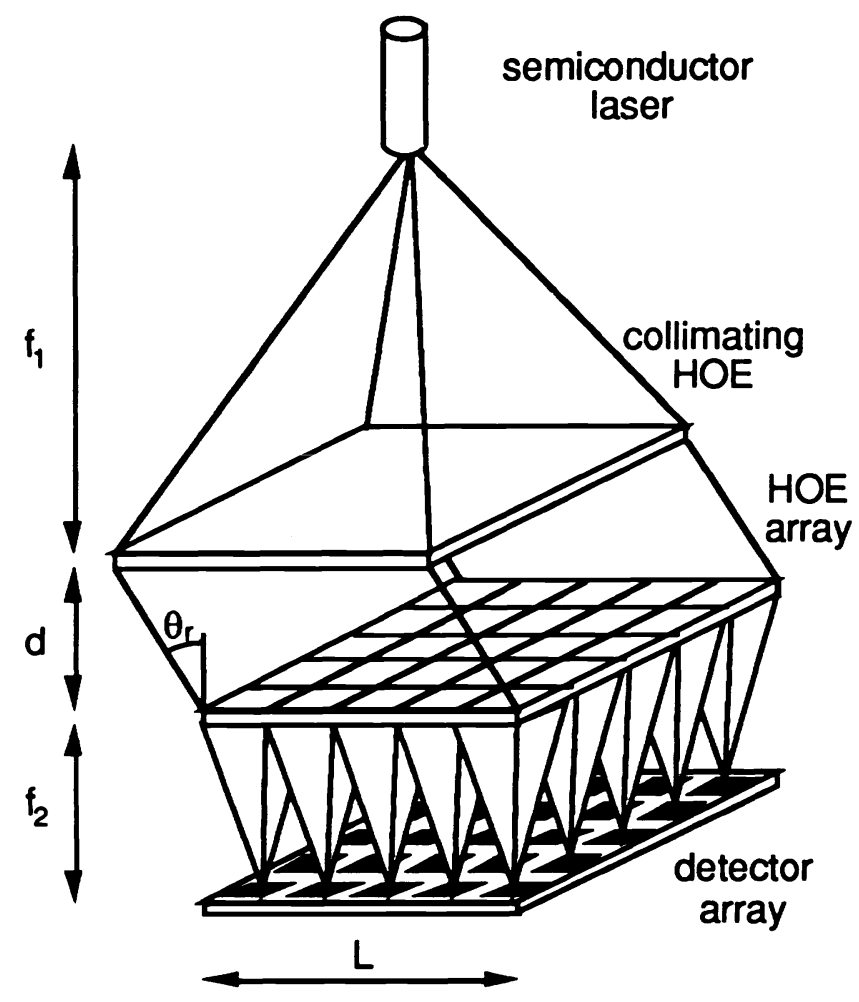

Fig.2 Doublet HOE consisting of a collimation $\mathrm{HOE}$ and a focusing HOE array.

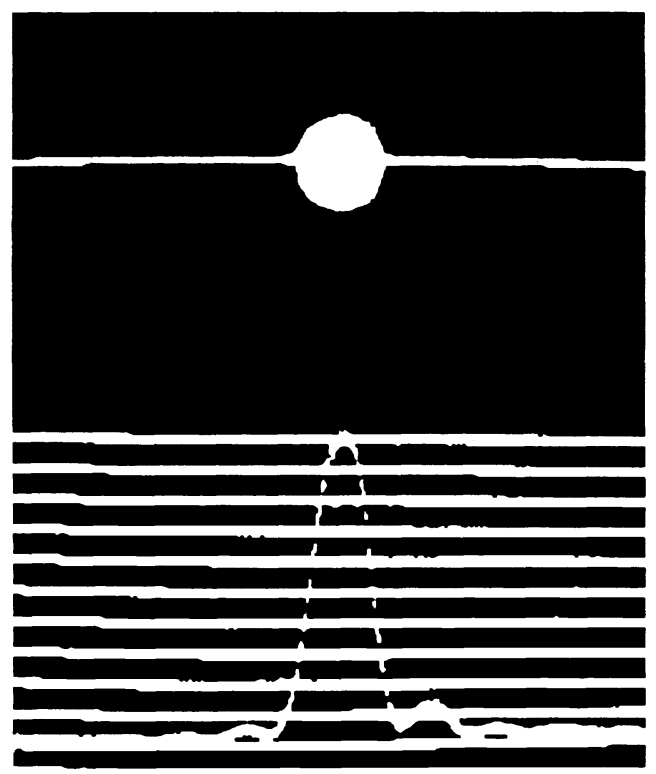

Fig.4 a) Center spot generated by the doublet HOE.

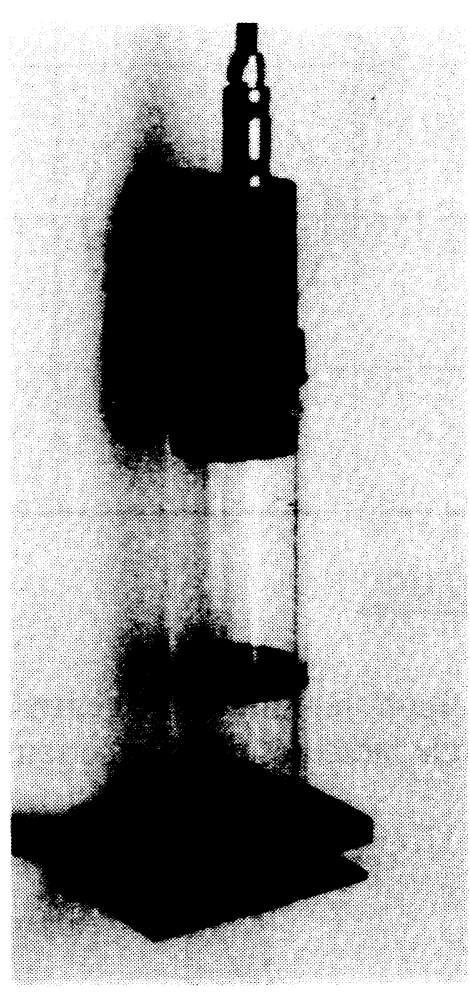

Fig.3 Laboratory prototype corresponding to Fig. 2.

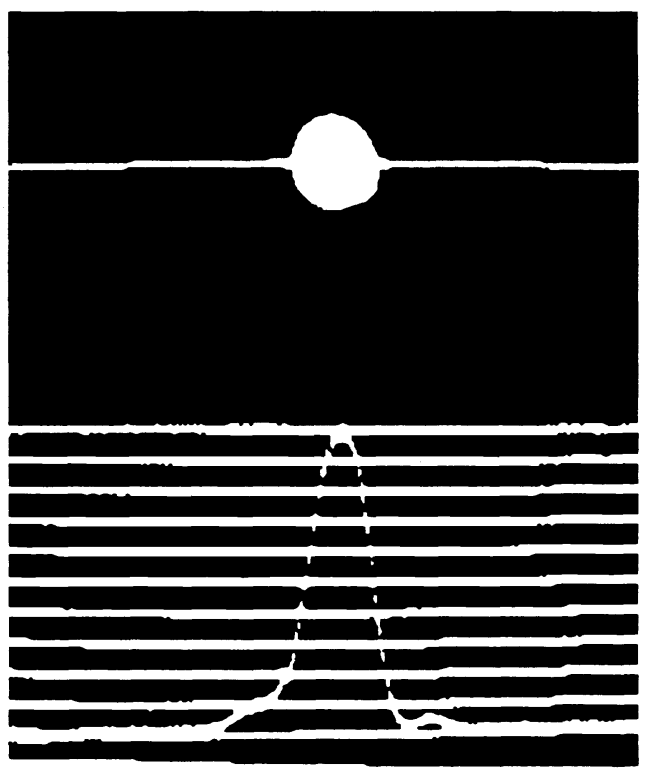

b) Corner spot generated by the doublet HOE. 


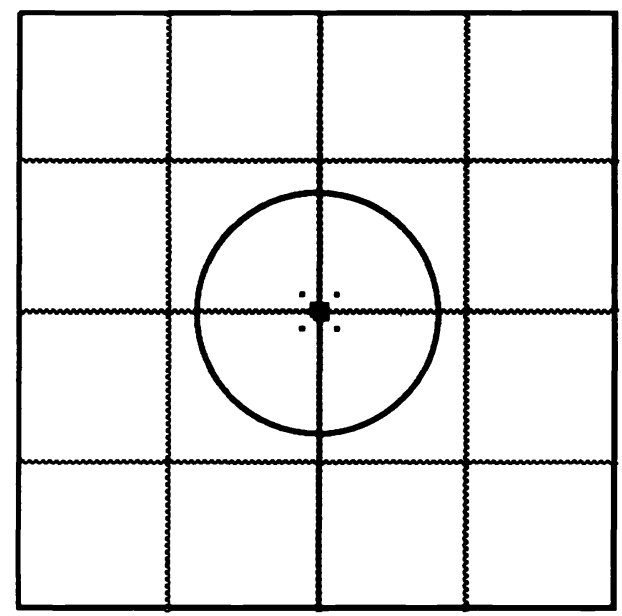

$10 \mu \mathrm{m}$

Fig.5 a) Spot diagram of the center spot. The circle indicates the diffraction limit.

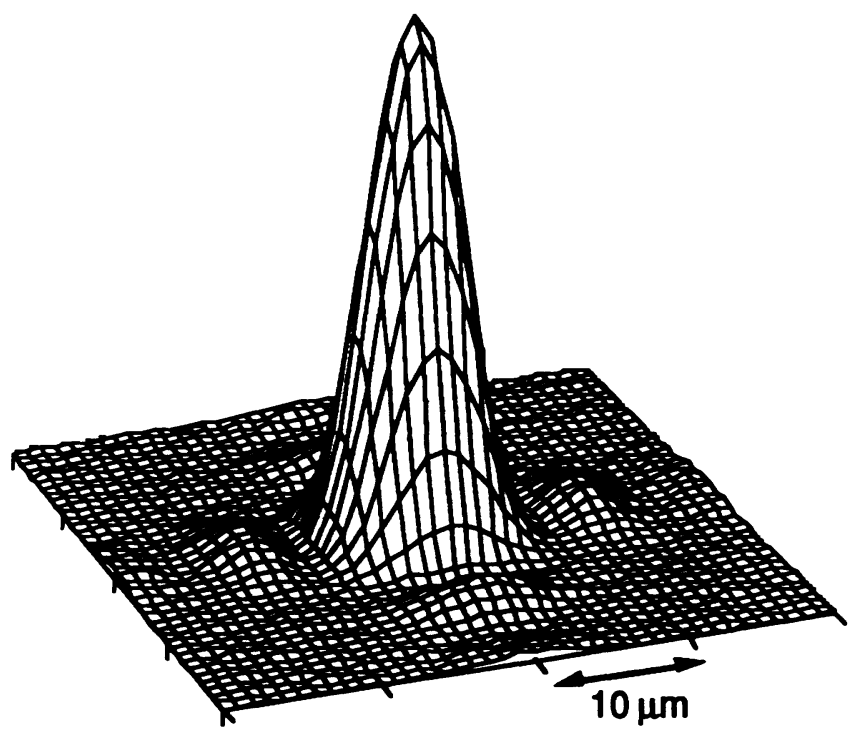

Fig.6 a) Beam profile of the center spot.

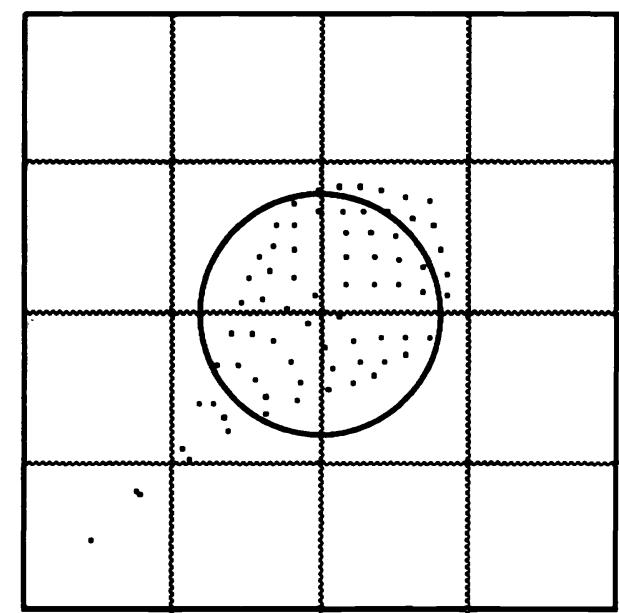

$10 \mu \mathrm{m}$

b) Spot diagram of the corner spot. The circle indicates the diffraction limit.

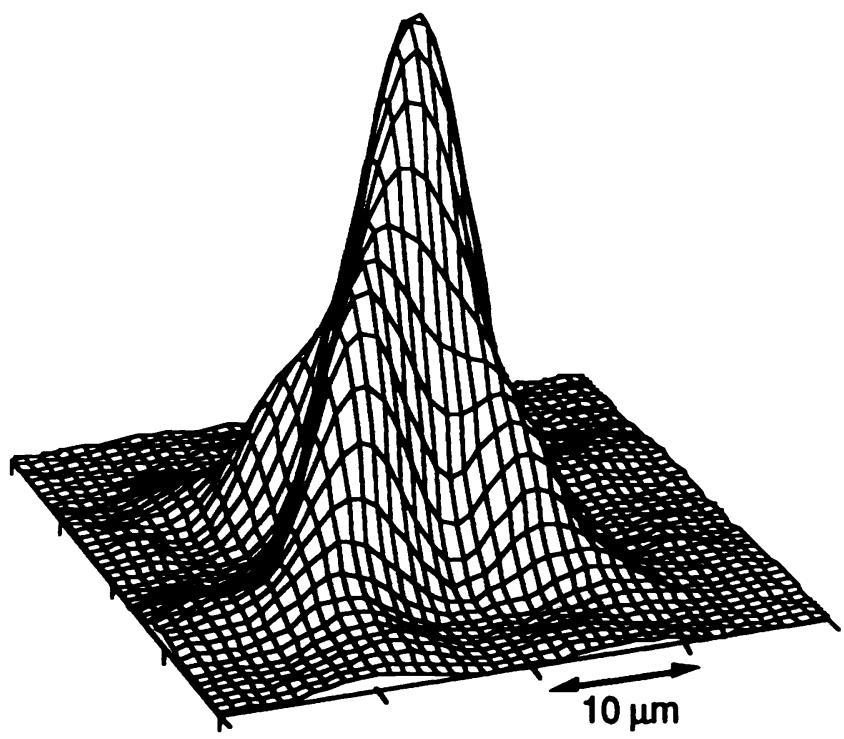

b) Beam profile of the corner spot.

\section{NEAR FIELD HOLOGRAPHY FOR INTERCONNECTION}

The prototype presented in the preceding chapter is of rather large size. Integrated circuits are much smaller. In this chapter we will show how miniaturized lenslet arrays with short focal lengths can be fabricated. 


\subsection{Principle}

Transmission holograms are recorded by interfering an object and a reference wave, where both sources have to be on the same side of the hologram. For recording holographic lenses with short focal lengths, a problem occurs by reasons of geometry. This is overcome by using total internal reflection (TIR) nearfield holography, as first reported by Stetson?. A mask is recorded holographically by passing a collimated beam through the mask, which is placed in close proximity (typically $50-500 \mu \mathrm{m}$ ) to the photosensitive layer (Fig.7). The transmitted wave interferes with the reference wave, which is fed through a prism and totally reflected at the film-air interface. An array of pinholes is fabricated as a chrome on glass mask by electron beam lithography. When illuminated with a plane wave, the mask generates by diffraction an array of spherical waves. This technique assures a position accuracy of about $0.2 \mu \mathrm{m}$ between the light spots. For the reconstruction without prism, the readout beam can be coupled into the hologram substrate by a small prism or another holographic grating, which acts also as collimator. This coupling element can be fabricated in the same film as the lenslet array. Collimator and lenslet array form together a compact system, as shown in Fig.8. Because of its symmetry, this system is quite insensitive to wavelength changes.

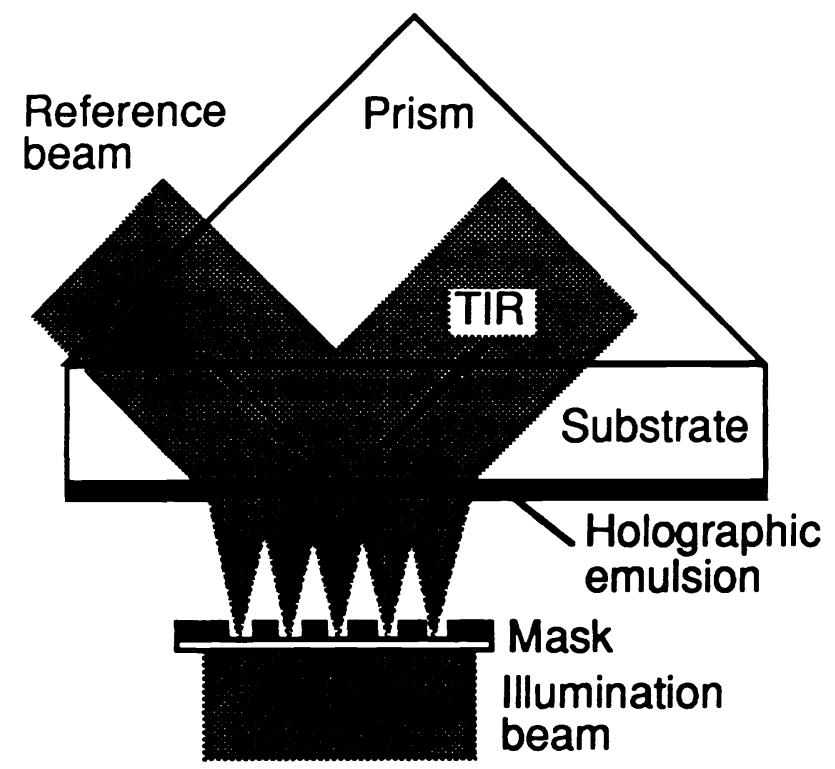

Fig.7 Near-field TIR recording geometry.

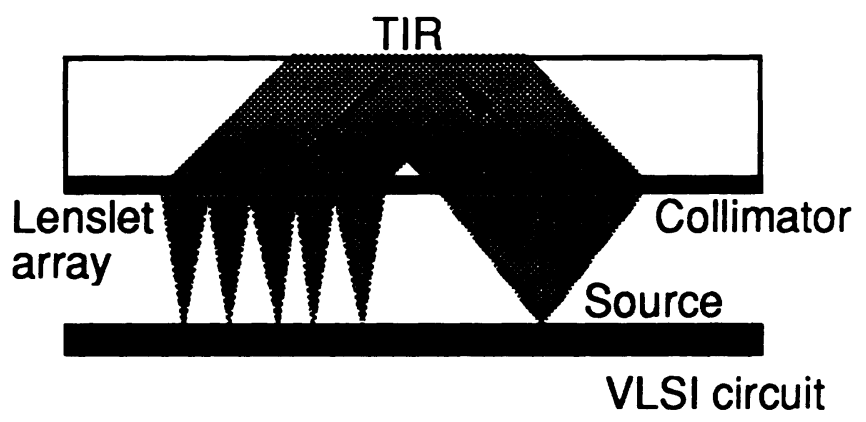

Fig.8 Compact system for near-field TIR doublet HOE.

\subsection{Lenslet array}

To test the performance of the TIR method, we have fabricated a $100 \times 100$ array of lenslets, equally distributed on a surface of $1 \mathrm{~cm}^{2}$, which corresponds to a spacing of $100 \mu \mathrm{m}$ between the spots. Each holographic lens has a focal length of $\mathrm{f}=390 \mu \mathrm{m}$, and a diffraction limited spot size of $\varnothing=10 \mu \mathrm{m}$. Figure 9 shows a part of the generated spot pattern. 


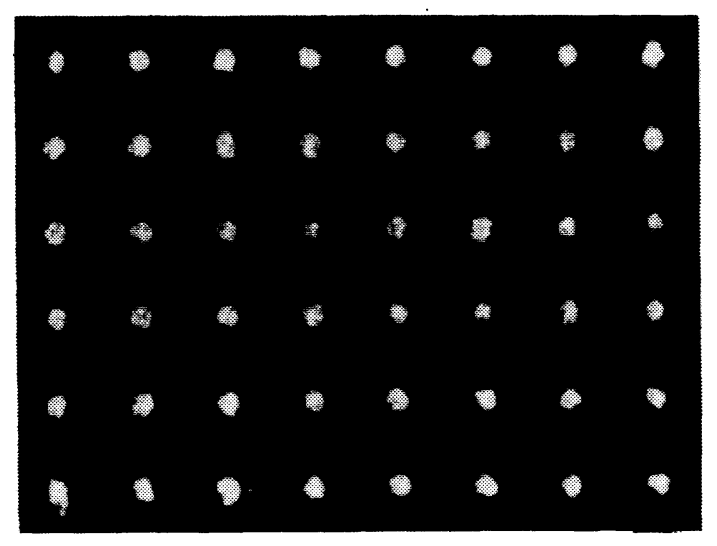

Fig.9 Partial image of the $100 \times 100$ lenslet array.

\subsection{Interconnection results}

Actually we apply the TIR method to fabricate a non-regular lenslet array for demonstrating clock distribution in an integrated circuit. We will give here some preliminary results.

For that purpose, a special test chip, shown in Fig.10, has been realized at the CSEM (Centre Suisse d'Electronique et de Microtechnique) using CMOS technology (CMN20A of VTI). Its outer dimensions are $3 \mathrm{~mm} \times 3 \mathrm{~mm}$. Several arrays of photodetectors with different sizes from $100 \mu \mathrm{m} \times 100 \mu \mathrm{m}$ down to $3 \mu \mathrm{m} \times 3 \mu \mathrm{m}$ are integrated on the chip. The chip contains also transimpedance amplifiers and output buffers to interface the photodiodes to the external analyzing system.

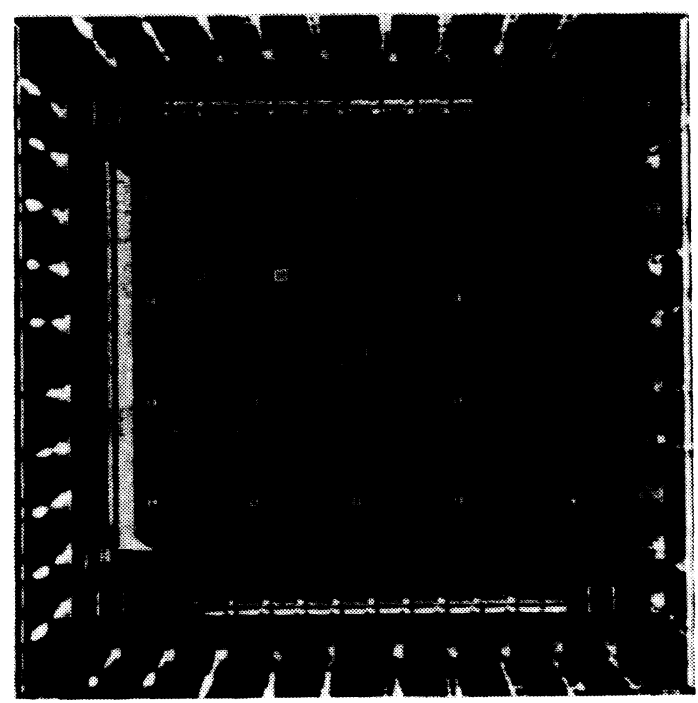

Fig.10 VLSI chip realized by the CSEM.

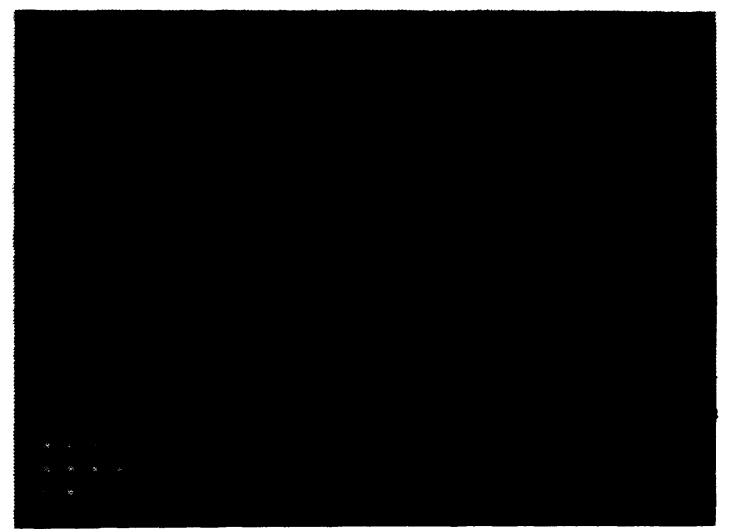

Fig.11 Partial image of the corresponding lenslet array.

The chrome mask to record the HOE has been fabricated by e-beam lithography. The detectors are now replaced by holes, where each hole acts as source when illuminated with a laser beam. The HOE has been 
recorded in a DuPont photopolymer. Figure 11 shows the reconstructed spot pattern at $\lambda=488 \mathrm{~nm}$. One can see that all patterns are reconstructed properly. It has been demonstrated recently ${ }^{8}$, that the TIR method is capable to reproduce structures down to $0.4 \mu \mathrm{m}$ for $\lambda=488 \mathrm{~nm}$.

\subsection{TIR method for the IR}

The TIR method requires a recording angle of typically $45^{\circ}$, which is larger than the angle for total reflection in glass. For large wavelength shifts between recording and reconstruction, the readout angle may then become larger than $90^{\circ}$. This is the case if the hologram is recorded at $\lambda=488 \mathrm{~nm}$ (dichromated gelatine, photopolymer) and reconstructed at $\lambda=780 \mathrm{~nm}$ (GaAlAs-laser). However, in that case the hologram can be recorded first at $\lambda=633 \mathrm{~nm}$ in a silver halide emulsion and then copied without the prism at $488 \mathrm{~nm}$ in dichromated gelatine (DCG) or photopolymer. In the copying process the Bragg angle can be readjusted, as reported in a previous paper ${ }^{1}$.

\section{DISCUSSION}

It has been shown with the doublet HOE configuration, that phase aberrations up to third order can be cancelled. Second order aberrations are eliminated by choosing the same angle for the image $\left(\theta_{\mathrm{P}}\right)$ and the object $\left(\theta_{\mathrm{O}}\right)$, third orders by choosing an on-axis set-up $\left(\theta_{\mathrm{P}}=\theta_{\mathrm{O}}=0^{\circ}\right)$. But, it is not possible to cancel all aberrations for the entire HOE if only plane and spherical wave fronts are used to record and replay the HOE. However, we could minimize the aberrations (all orders together) by optimizing the recording geometry, using a ray-tracing program and the root mean square radius as criteria.

The Bragg condition and the condition for low aberrations are only fulfilled rigorously near the center of the HOE. Since in our case the aperture of the focusing facets are small, the spots are nearly diffraction limited and the Bragg mismatch at the borders of the HOE is small enough to give nearly optimum diffraction efficiency. Note that, for the same numerical aperture (NA), the aberrations decrease with smaller system size, i.e. with shorter focal length.

For higher numerical apertures, the aberrations will certainly prevail over the diffraction limit and determine the spot sizes. Then, it will be very important to minimize all aberrations. The Bragg mismatch will become significant at the borders of the HOE and the HOE diffraction efficiency will decrease. In this case, aspherical wave fronts for recording the HOE must be used to minimize the aberrations and to fulfill the Bragg condition simultaneously. These wave fronts can be provided by computer generated holograms $(\mathrm{CGH})^{5}$.

The automatic step and repeat system for the recording of holographic lenslet arrays can provide a nearly unlimited number of focal spots. In addition, the generated pattern needs not to be regular. The light intensity of each spot can be controlled by the aperture during recording, which determines also the spot size. The TIR holography is very powerful to record very large numbers of regularly spaced facets with very short focal lengths. The numerical aperture of the pinholes and the distance between mask and HOE have to be appropriately chosen to avoid large overlapping of the diffraction patterns, which would cause intermodulations.

Compared with computer generated lenslet arrays ${ }^{9}$, the holographic method (recording by interference) has the advantage that holographic materials with very high resolution can be used. Recording gratings with more than 2000 lines/mm is standard for materials such as DCG, photopolymers and photoresist. 


\section{CONCLUSION}

A prototype of a $5 \times 5$ holographic lens array operating at $\lambda=780 \mathrm{~nm}$ has been fabricated with a doublet HOE. The doublet HOE is free of aberrations up to third order and works under Bragg condition. It would perfectly feed a $5 \times 5$ array of $10 \times 10 \mu \mathrm{m}^{2}$ large detectors. The recording geometry is simple and a step and repeat process is used for the array.

We have investigated the near-field total internal reflection (TIR) holographic recording technique to solve the problems of miniaturization. With this method a holographic lenslet array of $10^{\prime} 000$ interconnection points with short focal lengths of $\mathrm{f}=390 \mu \mathrm{m}$ and spot sizes of $10 \mu \mathrm{m}$ has been fabricated successfully.

Finally, the TIR method has been applied to the clock distribution on a specially designed VLSI circuit.

\section{REFERENCES}

1. D. Prongue, H. P. Herzig, "Design and fabrication of HOE for clock distribution in integrated circuits," Proc. of the International Conference on Holographic Systems, IEE Publication No. 311, pp. 204-208, 1989.

2. H. P. Herzig, R. Dändliker, "Holographic optical scanning elements: analytical method for determining the phase function", J. Opt. soc. Am. A, 4, pp. 1063-1069, 1987.

3. H. P. Herzig, "Holographic optical elements (HOE) for semiconductor lasers", Opt. commun., 58, pp. 144-148, 1986.

4. M. Assenheimer, Y. Amitai and A. A. Friesem, "Recursive design for an efficient HOE with different recording and readout wavelength", Appl. Opt., 27, pp. 4747-4752, 1988.

5. H. Buczek, J. M. Teijido, "Application of electron-beam lithography at CSEM for fabricating computer-generated holograms", SPIE 884, pp. 46-51, 1988.

6. H. Kogelnik, "Coupled wave theory for thick hologram gratings", Bell. Syst. Tech. J., 48, pp. 2909-2947, 1969.

7. K. Stetson, "Holography with total internally reflected light", Appl. Phys. Lett., 11, pp. 225-226, 1967.

8. R. Dändliker, J. Brook, "Holographic photolithography for submicron VLSI structures", Proc. of the International Conference on Holographic Systems, IEE Publication No. 311, pp. 127-132, 1989.

9. M. R. Feldman, C. C. Guest, "Computer generated holographic optical elements for optical interconnection of very large scale integrated circuits", Appl. Opt., 26, pp. 4377-4384, 1987. 